\title{
Parto e idade: características maternas do estado do Rio Grande do Sul
}

\author{
Juliana Falcão Padilha* Rita Patrícia Pereira Torres** Andriele \\ Gasparetto $^{\star * *}$ Lourdes Boufleur Farinha ${ }^{* \star *}$ Karen de Mello \\ Mattos $^{* * * *}$
}

Resumo: Objetivo - Analisar o tipo de parto e idade da mãe em relação ao número de nascimentos no RS em uma série histórica. Métodos - pesquisa descritiva, realizada de janeiro a julho de 2012 na base de dados DATASUS de 2005 a 2010. Resultados - evidenciou-se uma maior prevalência de partos cesáreos, com índice estimado de $58,09 \%$, em 2010 . Outro ponto a ser ressaltado foi em relação a faixa etária, onde em 2010, mulheres de 20-29 anos e 30-39 anos atingiram 49,29\% e $31,13 \%$ das ocorrências, respectivamente. Conclusão - Apesar de campanhas destacarem os benefícios do parto vaginal, evidencia-se neste estudo, um significativo aumento do número de parto cesáreo, bem como da ocorrência de gestações em idade mais avançada. Porém, se faz necessário mais estudos epidemiológicos para que se possam instrumentalizar ações em saúde voltadas para a situação avaliada e um maior aproveitamento de dados disponibilizados pelos sistemas de informações.

Descritores: Parto Obstétrico; Idade Materna; Cesárea; Nascimento Vivo.

\section{Birth and age: maternal characteristics of Rio Grande do Sul State}

Abstract: Objective - Analyze the type of birth and mother's age related to numbers of births in the state in a historical series. Methods - descriptive researches realized from January to July 2012 on DATASUS data basis between 2005-2010. Results - it was pointed a greater prevalence of cesarean birth with estimated index of $58.09 \%$ on 2010 . Other point to highlight was related to the age group, where in the same year, 2010, women of 20-29 years and $30-39$ years old reached $49.29 \%$ and $31.13 \%$ of the occurrences, respectively. Conclusion - despite of campaigns highlighting the benefits of vaginal birth, it is pointed in this study a significant increase of the number of cesarean birth, as well as the occurrences of gestation on advanced age. However it is needed more epidemiological studies to enable actions on health with emphasis to the evaluated situation and a greater use of the available data by the information systems.

Descriptors: Delivery; Obstetric; Maternal Age; Cesarean Section; Live Birth.

\footnotetext{
*Mestranda em Fisioterapia na Universidade do Estado de Santa Catarina (UDESC), Florianópolis, SC, Brasil.

${ }^{* *}$ Graduanda em Enfermagem no Centro Universitário Franciscano (UNIFRA), Santa Maria, RS, Brasil.

*** Mestre em Ciências da Saúde pela Universidade Federal do Rio Grande (FURG), Rio Grande, RS, Brasil.

****Especializada em Enfermagem Médico-ciúrgica pela Faculdade de Enfermagem Nossa Senhora Medianeira (FACEM), Santa Maria, RS, Brasil.

${ }^{* * \star * \star}$ Mestre em Saúde Coletiva pela Universidade Luterana do Brasil (ULBRA), Santa Maria, RS, Brasil.
} 


\section{Introdução}

O número de nascidos vivos constitui-se em relevante informação para o campo da Saúde Pública, pois a partir do mesmo podem-se construir inúmeros indicadores voltados para avaliação de riscos à saúde do segmento materno-infantil1 .

Com base nisto, houve a necessidade da implantação de um sistema de informações específico que pudesse coletar dados sobre os nascidos vivos, pois estes só eram conhecidos a partir de informações censitárias². Em 1990 cria-se o Sistema de Informações de Nascidos Vivos (SINASC) que busca subsidiar as intervenções relacionadas à saúde da mulher e da criança para todos os níveis de atenção do Sistema Único de Saúde (SUS), com ações direcionadas à gestante e ao recém-nascido. $O$ acompanhamento da evolução das séries históricas do SINASC permite a identificação de prioridades de intervenção, o que contribui para efetiva melhoria do sistema ${ }^{3}$.

A área técnica de Saúde da Mulher é responsável pelas ações de assistência ao prénatal, incentivo ao parto natural e redução do número de cesáreas desnecessárias, redução da mortalidade materna, dentre outras ${ }^{4}$. Assim, para a saúde materna e neonatal, é fundamental que se busque uma atenção de qualidade e de forma humanizada. A atenção à mulher na gestação e no pós-parto deve incluir ações de prevenção e promoção da saúde, além de diagnóstico e tratamento adequado dos problemas que ocorrem neste período ${ }^{5}$.

Para atender às necessidades desse segmento, é necessário que o governo federal, por meio do Ministério da Saúde (MS), assim como os estados e municípios desenvolvam estratégias com o objetivo de organizar os sistemas de atenção à gestação, parto e puerpério, visando uma assistência hierarquizada e integralizada no sentido de cumprir os princípios constitucionais do SUS6.

Nos países em desenvolvimento, particularmente no Brasil, a alta incidência de cesariana desnecessária, ou seja, aquela que ocorre sem indicação, é preocupante, o que se caracteriza como um grave problema de Saúde Pública ${ }^{7}$. Tendo em vista este aumento, o MS criou diferentes políticas e estratégias de saúde para tentar diminuir esse crescimento, como a Política Nacional pelo Parto Natural e Contra as Cesáreas Desnecessárias (2008), a Rede Cegonha (2011) dentre outras ${ }^{8}$.

Segundo dados do último Censo (2010), fornecidos pelo Instituto Brasileiro de Estatística e Geografia (IBGE), o Rio Grande do Sul possui 496 municípios e 10.693.929 habitantes, sendo que 5.488.872 são do sexo feminino e aproximadamente 2.917 .920 estão em idade fértil| .

Tendo como premissa a relevância e o impacto da saúde materno-infantil na qualidade de vida desta população e a importância do acompanhamento dos indicadores referentes ao período gestacional e nascimento, faz-se necessário investigar a situação do Estado do Rio Grande do Sul em relação às variáveis maternas, a fim de detectar pontos negativos e positivos na rede pública de assistência à gestante, parturiente e puérpera, para que possa ser efetuado um panorama desta população com posterior elaboração de ações das entidades competentes. Assim, o objetivo desta pesquisa foi analisar o tipo de parto e a variável idade da mãe em relação ao número de nascimentos no Estado do Rio Grande do

Saúde (Santa Maria), v.39, n.2, p.99-108, 2013. Parto e idade: Características maternas do estado do Rio Grande do Sul Sul em uma série histórica. 


\section{Metodologia}

Este estudo caracteriza-se como descritivo com abordagem quantitativa, sendo a coleta de dados realizada por meio de dados secundários obtidos na base do DATASUS/ INFORMAÇÕES DE SAÚDE/ ESTATÍSTICAS VITAIS (disponível online no sistema TABNET do Rio Grande do Sul, com possibilidade de realizar cópias das tabelas em formato CVS), no período de janeiro a julho de 2012.

As variáveis analisadas para esta pesquisa foram: número de nascimentos, tipo de parto e idade da mãe. Porém, no site do DATASUS encontram-se outras variáveis, não abordadas neste trabalho, como grau de instrução da mãe, Apgar no $1^{\circ}$ e $5^{\circ}$ minuto, peso ao nascer, dentre outros. As variáveis analisadas e o período escolhido (2005 a 2010) foram selecionados a partir da necessidade local, conforme determinação da equipe epidemiológica da $4^{\circ}$ Coordenadoria Regional de Saúde.

Para análise estatística, os dados coletados foram organizados em planilhas do Programa Microsoft Excel versão 2007, utilizando estatística descritiva simples, apresentando tanto os valores absolutos como seus respectivos percentuais.

Cabe salientar, que estas informações foram utilizadas exclusivamente para alcançar os objetivos do estudo, tomando o cuidado de manter o sigilo necessário, bem como evitar qualquer prejuízos as pessoas ou instituições envolvidas.

\section{Resultados e Discussão}

A partir deste estudo observou-se que o total de partos ocorridos entre 2005 e 2010 foi de 823609 (excluindo os partos do tipo ignorado - 360), sendo $386.408(46,91 \%)$ partos do tipo vaginal e 437.201 (53,08\%) cesáreo. Somente no ano de 2010, ocorreram 133.135 partos (novamente excluindo os ignorados - 108) com um total de $77.344(58,09 \%)$ cesáreos e 55.791 (41,90\%) vaginais. Observa-se que o parto vaginal no Estado do RS está diminuindo progressivamente em relação ao cesáreo com o passar dos anos. No ano de 2006, o parto cesáreo $(50,50 \%)$ era equivalente ao vaginal $(49,47 \%)$, no entanto, o parto cesáreo passou a se manter em destaque a partir de 2007. Ressalta-se então, que apesar de ter ocorrido um decréscimo de parto cesáreo entre 2005 e 2007, o número de partos vaginais encontra-se em constante declínio, desde 2005, (FIGURA 1).

Figura 1 - Número de nascimentos por Tipo de Parto. Rio Grande do Sul, 2012. Fonte: DATASUS, 2012.32

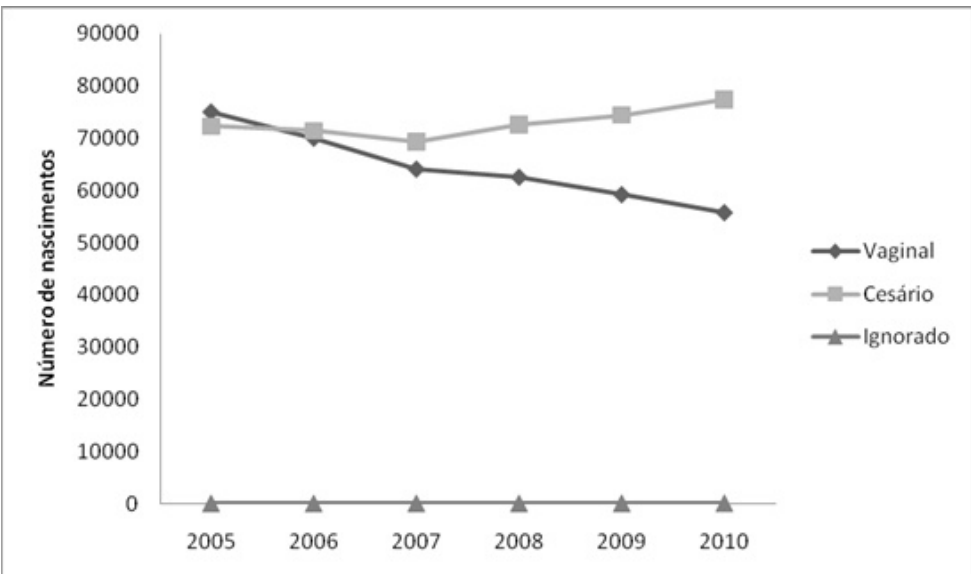

Rev. Saúde (Santa Maria), Santa Maria, v.39, n.2, p.99-108, Jul./Dez.2013. Padilha, J. F; et al. 
Dentro deste contexto, em 2010 , relatou-se que $58,09 \%$ dos partos foram cesáreos, excedendo o preconizado pela Organização Mundial de Saúde (OMS) que é de 15\%.

Entre os fatores que influenciam a alta incidência de parto cesáreo estão: acesso a informação e serviços de saúde, fatores culturais ${ }^{10}$, variáveis socioeconômicas, escolaridade, raça, dentre outros ${ }^{11}$, como a alusão ao afastamento da dor e do sofrimento. Neste sentido, é relevante que os profissionais da área da saúde estejam comprometidos em campanhas esclarecendo as vantagens e desvantagens dos variados tipos de partos. Ainda, é importante identificar as necessidades de cada gestante, para contribuir na escolha consciente do tipo de parto mais adequado a cada uma delas, e assim proporcionar conforto, qualidade no atendimento, bem como informações e esclarecimentos adequados ${ }^{12}$.

O parto cesáreo demanda um tempo de recuperação maior em comparação ao vaginal, expondo a gestante a processos infecciosos ou hemorrágicos ${ }^{11}$. Somado a isso, deve-se considerar que por ser um procedimento cirúrgico, deve ser realizado exclusivamente em âmbito hospitalar e com acompanhamento de profissionais habilitados ${ }^{13}$, não podendo ser excluído o risco de laceração de algum órgão, problemas de cicatrização e que sua frequência limita o número de partos ${ }^{14}$.

Em contrapartida, o parto vaginal proporciona tanto para a mãe como para o recém nascido inúmeros benefícios, como rápida recuperação, diminuição dos riscos de processos infecciosos, além de proporcionar grande vínculo entre mãe e filho ${ }^{14}$. Yazalle e colaboradores (2001) realizaram uma análise retrospectiva dos partos ocorridos no município de Ribeirão Preto-SP, no período de 1986-1995. Evidenciou-se que a incidência de cesáreas está relacionada com o nível social das mulheres, assim, quanto mais cultas forem estas mulheres, maior o número de cesáreas, sendo inversamente proporcional ao risco da gestação, ou seja, incidência maior de cesáreas nos grupos de menor risco e de menor incidência de doenças ${ }^{15}$.

Dados do SINASC de 2009 mostram que a taxa brasileira de partos cesáreos e vaginais são de 50,1\% e 49,9\%, respectivamente, atingindo índices de 44\% para vaginais e 55,99\% cesáreos na região Sul. O Centro-Oeste possui índices semelhantes ao Sudeste sendo respectivamente $44,17 \%$ vaginais, $55,82 \%$ cesáreos; $43,23 \%$ vaginais e $56,76 \%$ cesáreos. Por fim, a região Nordeste possui $58,73 \%$ das resoluções dos partos pelo tipo vaginal e $41,26 \%$ pelo tipo cesáreo, sendo a segunda região com maior ocorrência de partos vaginais, perdendo apenas para a Norte com $60,36 \%$ vaginais e $39,42 \%$ de cesáreos ${ }^{16}$.

Ao analisar o tipo de parto por Unidade de Federação, dois estados obtiveram destaque: o Amapá, que dos 14.248 nascimentos no ano de 2009, 29,09\% foram do tipo cesáreo e 70,90\% vaginal; e Roraima, com 26.053 partos neste mesmo ano, sendo o maior índice de cesáreas do Brasil, atingindo $61,24 \%$ e com consequente menor ocorrência de parto vaginal $(38,75 \%) 16$.

No entanto, é importante ressaltar e evidenciar o elevado e crescente índice de parto cesariano no Rio Grande do Sul. Com os avanços tecnológicos na obstetrícia nas últimas décadas, o parto passou a ser visto quase como uma patologia e a parturiente como uma paciente, não participando ativamente do processo do nascimento. Processo esse cada vez menos natural, a tal ponto que atualmente no Brasil existe um movimento governamental no sentido da humanização da assistência obstétrica ${ }^{17}$.

A atenção humanizada ao parto e nascimento é fundamentada na importância do fortalecimento do protagonismo e da autonomia da mulher neste momento, esta participa nas decisões referentes às condutas. Este tipo de atenção humanizada protege a mulher contra 
violência ou negligência, reconhece os direitos fundamentais delas e das crianças a tecnologias apropriadas de atenção em saúde, com a adoção de práticas baseadas em evidências, e garante o direito à acompanhante de livre escolha ${ }^{18}$.

Desde 2006, o Ministério da Saúde promove a Campanha Nacional de Incentivo ao Parto Normal e Redução da Cesárea Desnecessária e em 2008 lançou a Política Nacional pelo Parto Natural e Contra as Cesáreas Desnecessárias, em parceria com a Agência Nacional de Saúde Suplementar (ANS) ${ }^{19}$.

0 aumento das taxas de parto cesáreo é um fenômeno que vem acontecendo no mundo todo, sendo que diversos países apresentam uma diminuição de partos vaginais no desfecho do nascimento. Estudo realizado de uma série histórica de 1991 a 2007 nos Estados Unidos mostrou que após um declínio no início de 1990, a taxa de cesarianas aumentou, marcando 2007 como $11^{\circ}$ ano consecutivo de aumento da taxa de cesária e constituindo alta recorde para o país. Estas taxas aumentaram modestamente de 1996-2000 para as mulheres em todos os grupos etários, sendo mais expressivas de 2000 a 2007 (33\% $)^{20,21}$.

Apesar da diferença entre os sistemas de saúde do Brasil e dos Estados Unidos, a taxa de cesariana neste último, constituiu o $12^{\circ}$ ano consecutivo de aumento no ano de 2008 (32,3\% de todos os nascimentos). Em 2009, novo recorde nas taxas de cesária com aumento de $32,9 \%$. Contrariando estas estimativas, dados preliminares de 2010 apontam para uma queda na taxa de cesariana de $32,8 \%$, a primeira em mais de uma década22,23,24,25, dados também encontrados no Brasil.

Em relação à Figura 2, que representa o número de nascimentos em relação a idade da mãe no período de 2005 a 2010, obteve-se 823.969 nascimentos, subdivididos em 5 grupos etários mais um grupo com dados ignorados. As gestantes de 10 a 14 anos totalizaram um número de $6.285(0,76 \%), 15$ a 19 anos 139.021 (16,87\%), 20 a 29 anos 408.512 (49,57\%), 30 a 39 anos $242.521(29,43 \%)$, mais de 40 anos $27538(3,34 \%)$ e dados ignorados completaram em $92(0,01 \%)$.

Somente no ano de 2010 ocorreram 133.226 nascimentos, excluindo os ignorados que foram de 17 , sendo que gestantes de 10 a 14 anos totalizaram $937(0,70 \%), 15$ a 19 anos 20.904 (15,69\%), 20 a 29 anos $65.680(49,29 \%), 30$ a 39 anos $41.477(31,13 \%)$ e mulheres acima de 40 anos, 4.228 (3,17\%) (FIGURA 2).

Figura 2 - Número de nascimento por Idade da Mãe. Rio Grande do Sul, 2012. Fonte: DATASUS, 2012.32

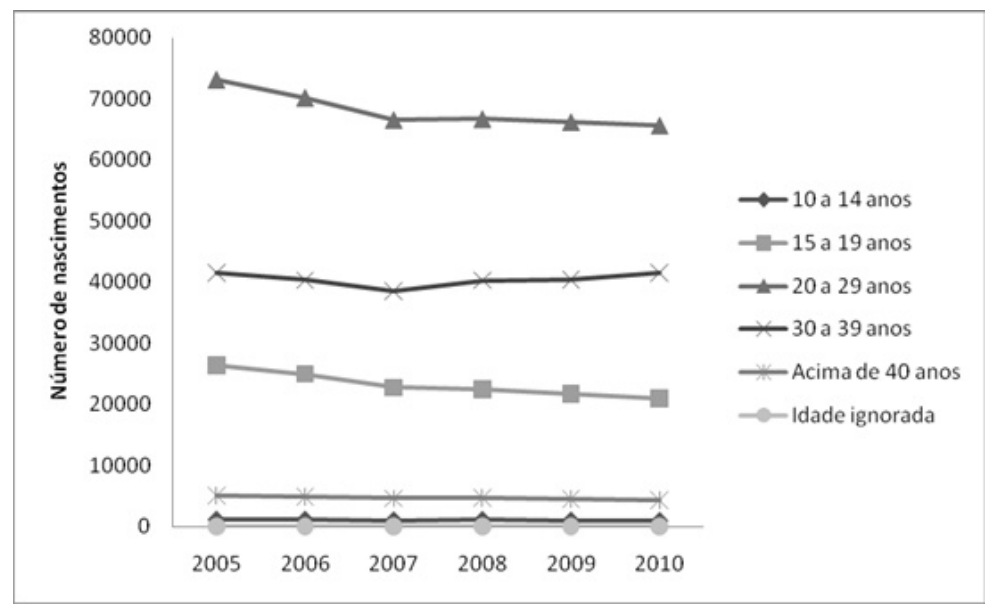

Rev. Saúde (Santa Maria), Santa Maria, v.39, n.2, p.99-108, Jul./Dez.2013. Padilha, J. F; et al. 
Assim, pode-se perceber que 0 índice de maior ocorrência de partos está na faixa etária de 20 a 29 anos, seguido pelas mulheres de 30 a 39 anos, adolescentes de 15 a 19 anos, mulheres acima de 40 anos e finalmente as pré-adolescentes de 10 a 14 anos (FIGURA2).

Além dos dados referentes ao tipo de parto, na análise deste estudo, observou-se um declínio no número de gravidez na adolescência, resultado este que deve ser mantido para evitar possíveis riscos que possam ocorrer as gestantes pertencentes a essa faixa etária. Alguns desses riscos são representados por aspectos materno-fetais, abandono do parceiro ou família, perda de unicidade com o grupo de iguais, descontinuidade e mesmo a interrupção de projetos de vida ${ }^{26}$, gerando alterações biopsicossociais inter-relacionados.

Apesar do grupo etário dos 10 aos 14 anos não apresentar grande percentagem no estado do RS, atingindo índice inferior a 1\%, representam um número importante de 6.285 ocorrências. A faixa de meninas de 10 a 14 anos, ainda não pertencente ao foco das políticas públicas voltadas para os direitos sexuais e reprodutivos, deve ser estudada para uma possível inserção nestes programas, com projetos desenhados de acordo com as necessidades e as especificidades deste grupo etário ${ }^{27}$.

Notou-se também um aumento crescente no número de gestantes com idade entre $30 \mathrm{e}$ 39 anos, a partir de 2007. Isso pode ser comprovado por estudos que afirmam que atualmente as mulheres estão buscando a maternidade por volta dos 35 anos, priorizando inicialmente a busca pela estabilidade profissional e emocional antes da gestação ${ }^{28}$. No entanto, após os 35 anos, faixa considerada pelos médicos como tardia para o início da gestação, devem ser considerados riscos significativos para o bom andamento da gravidez, os quais se destacam a hipertensão, a diabetes gestacional, a maior probabilidade de abortos espontâneos, partos prematuros, entre outros. Alguns autores ainda tendem a priorizar a variável idade que, isoladamente, é vista como responsável por problemas maternos e fetais ${ }^{29}$.

A partir de pesquisas realizadas, as mulheres mais velhas tornam-se mais propensas a complicações perinatais que são associadas às complicações caracteristicas do envelhecimento. Mulheres com faixa etária de 35 anos ou mais, apresentam um risco aumentado de anomalias cromossômicas, algumas destas podendo ser diagnosticados no pré-natal. Já, idade superior a 40 anos é um fator de risco independente para placenta prévia, descolamento prematuro da placenta, parto cesárea e mortalidade perinatal30. 0 papel da vigilância pré-natal de rotina em mulheres com idade de 40 anos ou mais, exige uma investigação mais aprofundada, pois essa população tem um risco aumentado de mortalidade perinatal, inclusive natimorto. Embora a probabilidade de efeitos adversos aumente com a idade materna, em geral, os resultados maternos e fetais são favoráveis nesta população ${ }^{30}$.

Gestantes na idade adulta são as que apresentam o maior índice de partos realizados, apresentando escores de até $68 \%$ dos nascimentos ${ }^{31}$, corroborando com os dados encontrados neste estudo, o qual encontrou-se um percentual de $82,35 \%$ para mulheres adultas com mais de 20 anos.

Saúde (Santa Maria), v.39, n.2, p.99-108, 2013. Parto e idade: Características maternas do estado do Rio Grande do Sul 


\section{Conclusão}

Segundo os resultados encontrados no presente estudo, em relação aos tipos de partos realizados no estado do RS, é passível de questionamento a grande diferença entre os resultados obtidos e o que é preconizado pelo sistema de saúde conforme informações colhidas no SINASC.

Nota-se que, apesar das campanhas do Governo Federal e Estadual direcionadas ao incentivo do parto vaginal, concordante com o que preconiza a OMS, isso não é evidenciado na prática conforme dados obtidos no SINASC, no qual prevalecem os partos cesáreos com significativo aumento. No ano de 2007 , houve um declínio no número de partos cesáreos em relação ao parto vaginal, neste sentido, seria de suma importância compreender os motivos da ocorrência deste declínio, resultado que pode ser utilizado como parâmetro para montagem de estratégias que consigam sensibilizar e esclarecer as gestantes sobre os benefícios e risco dos diferentes tipos de parto citados nesta pesquisa.

Além disso, atualmente, a mulher por estar inserida no campo de trabalho, mais independente financeira e emocionalmente e, ainda, considerando 0 avanço dos métodos contraceptivos, tem retardado $o$ início da gestação, fazendo com que se elevem os números de gestantes na idade adulta (30- 39 anos e mais de 40).

Deve-se, portanto, destinar maior atenção a população estudada no sentido de trazer informações que possam delinear a melhor escolha do tipo de parto a ser realizado. Os profissionais da saúde, baseados nas políticas públicas de saúde brasileira, devem, cada vez mais, se comprometerem com a assistência á mulher de maneira humanizada e competente durante 0 trabalho de parto e parto, buscando o bem-estar, saúde e atenção integral do público feminino com base em ações na promoção, prevenção, educação em saúde, e tratamento, sempre baseados em estudos epidemiológicos. Com isso, esses estudos permitirão a elaboração de ações de vigilância em saúde para que os pontos frágeis sejam melhorados e os pontos fortes cada vez mais aprimorados, assim garantindo uma assistência integral e de qualidade para a população feminina brasileira.

Durante a execução dessa pesquisa, algumas dificuldades e limitações foram sentidas, como a não inclusão de dados nos anos anteriores a 2005 e de outras variáveis em todas as regionais de saúde. Desta forma, sugere-se a realização de novas pesquisas que englobem as variáveis não analisadas e os anos não contemplados neste estudo, a fim de possibilitar o delineamento preciso do perfil dessa população e desta forma, contribuir de forma efetiva na elaboração de ações em saúde.

\section{Referências Bibliográficas}

1. Ministério da Saúde (BR). Guia de Vigilância Epidemiológica. $7^{a}$ ed. Brasilia (DF): Ministério da Saúde; 2009.

2. Ministério da Saúde (BR). Guia de Vigilância Epidemiológica. $6^{\text {a }}$ ed. Brasília (DF): Ministério da Saúde; 2005.

3. Portal da saúde - SUS: Sistema de Informações sobre Nascidos Vivos - SINASC [homepage]. Brasilia Rev. Saúde (santa Maria, santa mara, v39, n.2, (DF); Ministério da Saúde; [acesso em 30 out.2011]. [1]. Disponível em: p.99-108, Jul.02.2013. http://portal.saude.gov.br/portal/saude/visualizar_texto.cfm?idtxt=21379. 
5. Ministério da Saúde (BR). Pré-Natal e Puerpério: Atenção Qualificada e Humanizada. Brasilia (DF): Ministério da Saúde; 2005.

6. Ministério da Saúde (BR). Gestação de Alto Risco - Manual Técnico. $5^{\text {a }}$.ed. Brasília (DF): Ministério da Saúde; 2010.

7. Amorin MMR, Souza ASR, Porto AMF. Indicações de cesariana baseadas em evidências: parte I. Rev. Femina. 2010 Ago; 38(8): 415-422.

8. Portal da saúde - SUS [homepage]. Brasilia (DF); Ministério da Saúde; [acessado em 15 jan.2012]. [1]. Disponivel em: http://portalsaude.saude.gov.br/portalsaude/index.cfm.

9. Instituto Brasileiro de Geografia e Estatística (IBGE): Estados: Rio Grande do Sul [homepage]. Brasilia (DF): Ministério do Planejamento, Orçamento e Gestão. [acessado em 15 nov.2011]. [3]. Disponível em: http://www.ibge.gov.br/estadosat/perfil.php?sigla=rs.

10. Camara MFB, Medeiros M, Barbosa MA. Fatores sócio-culturais que influenciam a alta incidência de cesáreas e os vazios da assistência de enfermagem. Rev. Eletr. Enf. [lnternet] 2000; 2(1). [acessado em: 22 de ago.2011]. Disponivel em: http://www.fen.ufg.br/revista/revista2_1/Cesarea.html.

11. Knupp VMAO, Melo ECP, Oliveira JB. Distribuição do Parto Vaginal e da Cesariana no Município do Rio de Janeiro o Período de 2001 a 2004. Esc. Anna Nery Rev. Enferm. 2008 Mar; 12(1):39-44.

12. Melchiori LE, Maia ACB, Bredariolli RN, Hory RI. Preferência de Gestantes pelo Parto Normal ou Cesariano. Interação psicol. 2009 Jan/Jun; 3(1):13-23.

13. Queiroz MVO, Silva NSJ, Jorge MSB, Moreira TMM. Incidência e características de Cesáreas e de partos normais: estudo em uma cidade no interior do Ceará. Rev. bras. enferm. 2005 Nov/Dez; 58(6):687-91.

14. Portal da saúde - SUS: Parto normal: mais segurança para a mãe e o bebê [homepage]. Brasilia (DF); Ministério da Saúde; [acessado em 16 ago.2011]. [1]. Disponível em: http://portal.saude.gov.br/saude/visualizar_texto.cfm?idtxt=20911.

15. Yazlle MEHD, Rocha JSY, Mendes MC, Patta MC, Marcolin AC, Azevedo GD. Incidência de cesáreas segundo fonte de financiamento da assistência ao parto. Rev. saúde pública. 2001;35(2):202-206.

16. DATASUS- Departamento de Informática do Sistema único de Saúde. Nascidos Vivos - Regiões Brasileiras [homepage]. Brasilia (DF): Ministério da Saúde [acessado em 20 dez. 2011]. [2]. Disponível em: http://tabnet.datasus.gov.br/cgi/tabcgi.exe?sinasc/cnv/nvuf.def.

17. Matei EM, Carvalho GM, Silva MBH, Merighi, MAB. Parto Humanizado: um direito a ser respeitado. Cadernos - Centro Universitário S. Camilo. 2003 Abr/Jun; 9(2):16-26.

18. Caviochioli S. Saúde amplia estrutura para realizar partos normais. Brasilia (DF): Ministério da Saúde; 2013. [acessado em 21 ago.2013]. [1]. Disponível em: http://portal.saude.gov.br/portal/aplicacoes/noticias/default.cfm?pg=dspDetalheNoticia\&id_area=1529\&CO_N OTICIA=14467.

19. Portal da saúde - SUS: Ministério da Saúde apóia humanização do parto. Brasília (DF); Ministério da Saúde; 2010. [acessado em 21 ago.2013]. [1]. Disponível em:http://portal.saude.gov.br/portal/aplicacoes/noticias/default.cfm?pg=dspDetalheNoticia\&id_area=124\&CO NOTICIA=11929

20. Menacker F, Hamilton BE. Recent Trends in Cesarean Delivery in the United States. NCHS Data Brief. 2010 Mar; (35):1-8.

21. HamiltonBE,MartinJA, VenturaSJ. Births: Preliminary Datafor2007.NVSR. 2009Mar;57(12):1-23.

22. Martin JA, Hamilton BE, Sutton PD, Ventura SJ, Mathews TJ, Kirmeyer S et al. Births: Final Data for 2008. NVSR. 2010 Dez; 59(1): 1-72.

Saúde (Santa Maria), v.39, n.2, p.99-108, 2013. Parto e idade: Características maternas do estado do Rio Grande do Sul

23. Martin JA, Hamilton BE, Sutton PD, Ventura SJ, Mathews TJ, Kirmeyer S et al. Births: Final Data for 2007. NVSR. 2010 Ago; 58(24):1-86. 
24. Martin JA, Hamilton BE, Sutton PD, Ventura SJ, Osterman MJK, Kirmeyer S et al. Births: Final Data for 2009. NVSR. 2011 Nov; 60(1):1-104.

25. Hamilton BE, Martin JA, Ventura SJ. Births: Preliminary Data for 2010. NVSR. 2011 Nov; 60(2):1-36.

26. Ministério da Saúde (BR). Manual Técnico - Pré-Natal e Puerpério: Atenção Qualificada e Humanizada.

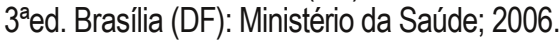

27. Costa TJNM, Heilborn ML. Gravidez na Adolescência e Fatores de Risco entre filhos de mulheres nas Faixas Etárias de 10 a 14 e 15 a 19 anos em Juiz de Fora MG. Rev. APS. 2006 Jan/Jun; 9(1): 29-38.

28. Zlotnik E, Segre CAM, Lippi UG. Índice de massa corpórea pré-gestacional, ganho ponderal e peso do recém-nascido de gestantes com idade igual ou superior a 35 anos. Einstein (São Paulo). 2007; 5(3):231238.

29. Gomes AG, Donelli TMS, Piccinini CA, Lopes RCS. Maternidade em Idade Avançada: Aspectos Teóricos e Empíricos. Interação psicol. 2008; 12(1):99-106.

30. Goldman JC, Malone FD, Vidaver J, Ball RH, Nyberg DA, Comstock CH et al. Impact of Maternal Age on Obstetric Outcome. Obstetrics \& Gynecology. 2005 May; 105(5) -part 1:983-990.

31. Santos GHN, Martins MG, Souza MS. Gravidez na adolescência e fatores associados com baixo peso ao nascer. Rev. bras. ginecol. obstet. 2008 Mai; 30(5):224-31.

32. DATASUS- Departamento de Informática do Sistema único de Saúde. Informações de Saúde [homepage]. Brasilia (DF): Ministério da Saúde. [acessado em 31 jul. 2012]. [2]. Disponível em: http://tabnet.datasus.gov.br/cgi/deftohtm.exe?sinasc/cnv/pnvrs.def

\section{Juliana Falcão Padilha}

Endereço para correspondência - Avenida Medianeira, 2020, apto. 04. Bairro Nossa Senhora de Lourdes, CEP: 97060-003, Santa Maria, RS, Brasil.

Currículo Lattes: http://lattes.cnpq.br/8493933674992420

E-mail: jufpadilha@gmail.com

Recebido em 24 de agosto de 2012.

Aprovado em 23 de setembro de 2013. 
Saúde (Santa Maria), v.39, n.2, p.99-108, 2013. Parto e idade: Características maternas do estado do

Rio Grande do Sul

108 | ISSN 2236-5834 